\title{
Cardiac tamponade secondary to right ventricular perforation caused by a temporary pacemaker lead in the course of myocardial infarction
}

\author{
Hiroki Shibutani, Kotaro Yutaka, Yu Mukai, Yuzo Akita, Masahiro Yoshinaga \\ Division of Cardiology, Osaka Saiseikai Izuo Hospital, Osaka, Japan
}

This case describes right ventricular (RV) perforation caused by a temporary pacemaker (TPM) lead during acute myocardial infarction (AMI). There is no consensus regarding the management of this complication.

A 67-year-old man with chest pain was admitted to the documented hospital. Electrocardiography revealed third-degree atrioventricular block and ST elevation in inferior leads (Fig. 1A).
Following insertion of a Swan-Ganz ${ }^{\mathrm{TM}}$ bipolar pacing catheter (Edwards Lifesciences Corporation, Irvine, CA, USA) in the RV, right coronary angiography revealed subtotal occlusion (Fig. 1B). Although atrioventricular block persisted, successful revascularization for inferior AMI reversed his status (Fig. 1C). Four hours postoperatively, his blood pressure gradually decreased with ventricular pacing failure (bradycardia followed by

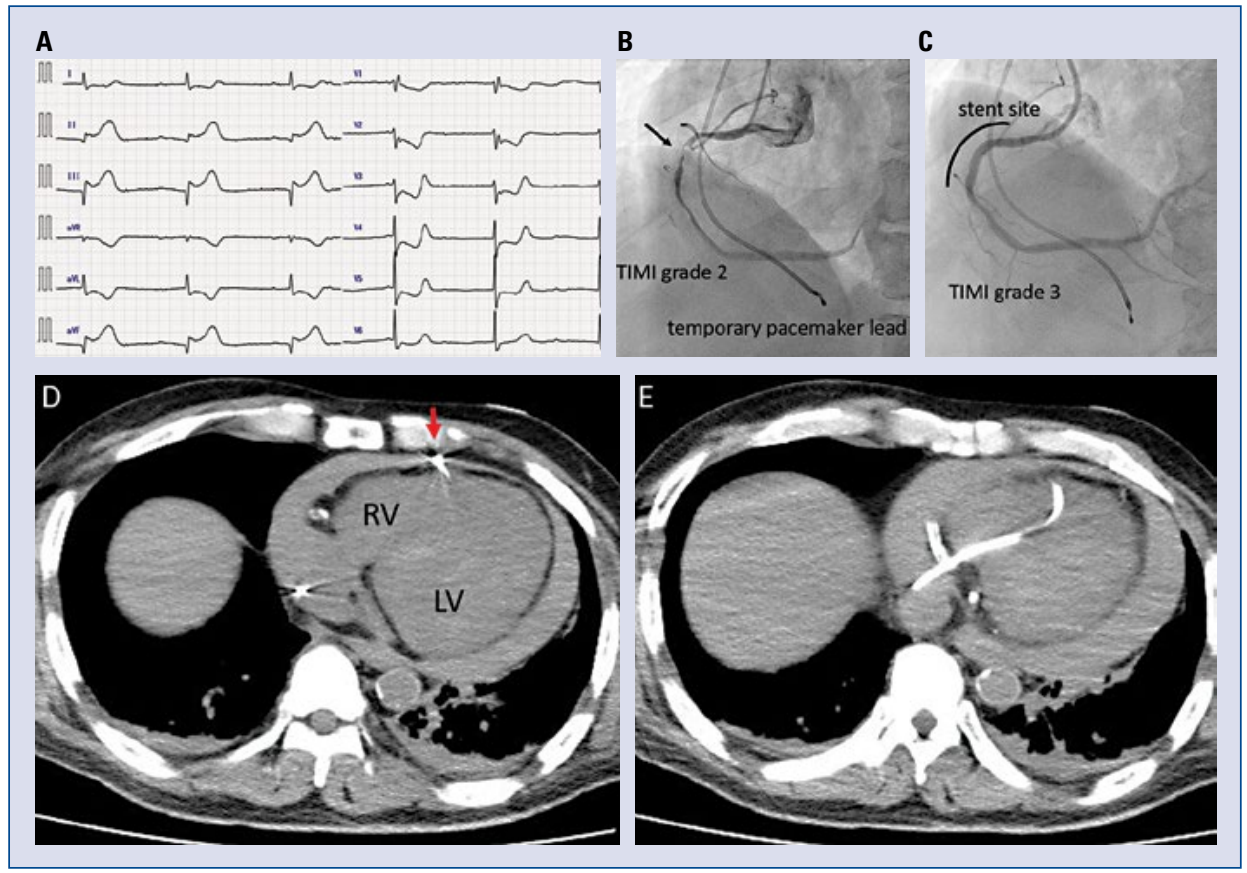

Figure 1. A. Electrocardiography on admission revealed complete atrioventricular block with escape rhythm and ST elevation in inferior leads. B. Baseline right coronary angiogram revealed thrombolysis in the myocardial infarction (TIMI), grade 2 flow. The black arrow indicates the culprit lesion. The temporary pacemaker lead was placed in the right ventricle (RV) via the right internal jugular vein. C. Post-intervention angiogram showed successful reperfusion and TIMI grade 3 flow. D, E. Autopsy imaging revealed severe pericardial effusion, with the temporary pacemaker lead visualized beyond the right ventricular wall (red arrow); LV — left ventricle.

Address for correspondence: Hiroki Shibutani, MD, Division of Cardiology, Osaka Saiseikai Izuo Hospital, 3-4-5 Kitayama, Taisho-ku, Osaka 551-0032, Japan, tel: +81-6-6552-0091, fax: +81-6-6552-0091, e-mail: hs.vxvii@gmail.com 
electromechanical dissociation), and he developed sudden cardiac arrest. This confirms the existence of a pericardial effusion using bedside echocardiography. Resuscitation efforts, including pericardiocentesis, were unsuccessful. The possibility that left ventricular free-wall rupture had occurred was considered; however, autopsy imaging revealed RV perforation caused by the TPM lead (Fig. 1D, E). The findings indicated that the cause of death was cardiac tamponade secondary to RV perforation. The TPM was removed after autopsy imaging.

Cardiac tamponade due to leakage from a low-pressure chamber, such as the RV, is observed less frequently. However, in thispatient, it was considered that myocardial infarction involving the RV and treatment with thrombolytic therapy (625 U of heparin per hour) and oral antiplatelet agents (acetylsalicylic acid $200 \mathrm{mg}$ and prasugrel $20 \mathrm{mg}$ ) were strongly associated with a weakened $\mathrm{RV}$ muscle and acute clinical course, resulting in tamponade. The only effective treatment was early detection and pericardiocentesis as a bridge to surgery. To prevent this situation, gentle catheter manipulation, including placement of the TPM on the intraventricular septum, was necessary.

\section{Acknowledgements}

We thank the staff of the Saiseikai Izuo Hospital. Furthermore, we are indebted to Editage, an editing company, for critical reading of the manuscript.

Conflict of interest: None declared 